\title{
Generic Structure and Rhetorical Relations of Online Book Reviews in English, Japanese and Chinese
}

\author{
Sonya Chik \\ Assistant Professor, Department of English, Kanagawa University, \\ Yokohama, Japan \\ sonyachik@kanagawa-u.ac.jp \\ Maite Taboada \\ Professor, Department of Linguistics, Simon Fraser University, British \\ Columbia, Canada \\ mtaboada@sfu.ca
}

\begin{abstract}
We examine the generic structure and rhetorical relations that characterise online book reviews in English, Japanese and Chinese to describe the pragmatic features of this emerging genre in a contrastive light. The corpus we analyse contains online book reviews written by consumers for consumers. The purpose of the study is twofold. First, we seek to identify the generic structure of online book reviews. Second, we investigate the cross-cultural variation in the rhetorical organisation of opinions and evaluations in written reviews across language communities. The reviews are analysed in terms of the generic stages of book reviews, which are determined by their overall communicative goals (Motta-Roth, 1995; Taboada, 2011). The stages are then mapped against rhetorical relations that capture the coherence and meaningful organisation of the text (Mann and Thompson, 1988). Results show that online book reviews in all three languages share a common generic structure comprising three broad stages: Metapragmatic Comment, Evaluation (Book Overall, Author, Plot, Character) and Recommendation. While Evaluation is the only obligatory stage, Metapragmatic Comment serves to prepare the reader for the Evaluation that follows. The recommendation stage is common in both English and Chinese reviews but is conspicuously absent in their Japanese counterpart. In terms of rhetorical patterns, Contrast, Concession and Antithesis relations are preferred in Metapragmatic Comment and Evaluation, while Motivation is typically present in the recommendation stage. This
\end{abstract}


paper proposes a methodology for the contrastive analyses of pragmatic phenomena, illustrating this methodology through the study of an emerging online genre.

\section{Keywords}

genre - generic stages - rhetorical relations - Rhetorical Structure Theory - online reviews - contrastive analysis

\section{Introduction}

Studies of pragmatic phenomena from a contrastive point of view occur at different levels of granularity, from the micro analyses of the realisation of speech acts (e.g., Blum-Kulka et al., 1989) or cohesive conjunctions (Kunz and Lapshinova-Koltunski, 2014) to the macro examination of conversational structure (Taboada, 2004) or application letters (Upton and Connor, 2001). We present a study in the macro area, which is aimed at understanding the generic structure and organisation of online book reviews in three languages, namely English, Japanese and Chinese.

The vast literature on contrastive studies and pragmatics provides a fruitful ground for our approach, which is inspired by Systemic Functional Linguistics and the concept of genre (Halliday and Hasan, 1985; Martin, 1984). We see genre as the first point of entry for any study of entire texts (text or talk), especially of the type presented here, an emerging genre. Genre in the sfu tradition enables the description and analysis of texts as the realisation of the context of culture of a specific language and society. Thus, a genre approach is particularly well suited to contrastive study and complements efforts, such as those of Matthiessen, to provide a cartography of registers in a language (Matthiessen, 2015). In SFL, genre is the realisation of the context of culture, while register encodes the context of situation. We focus here on the context of culture, as a means for understanding the similarities and differences of the emerging online book review genre across languages. As we detail in Section 3, genre analysis involves identifying the purpose of the genre and the stages in which it develops. This is of particular interest in an online genre, where conventions may be emerging and may be created by the communities that produce texts in the genre in question. Our research question, then, is whether three different language communities enact the online book review genre in similar or different ways.

The other level of organisation that we are interested in is the rhetorical structure of texts, in terms of relations across propositions, as proposed 
in Rhetorical Structure Theory (Mann and Thompson, 1988). This approach allows for a closer examination of the type of relations that are present in texts, and their location with regard to generic stages. The research question here is similar: Do the three languages employ different rhetorical relations, at different rates or in different stages within the texts?

Taken together, these two analyses constitute a productive methodology by which to approach a genre from a contrastive point of view. We will see, in the analysis and results sections, that we are able to highlight how the languages construct texts in very similar ways from a generic point of view, but at the same time showing differences that may be the result of cultural practices and the influence of other genres in each language.

This paper is organised as follows. In Section 2, we provide a necessarily brief survey of the tenets of our approach: the review genre, contrastive analyses and the SFL approach to contrastive studies. In Section 3, we describe the data and methodology used, while Section 4 provides the results of the analysis. We conclude Section 4 with some implications for contrastive pragmatics, providing general conclusions in Section 5 .

\section{The Review Genre}

Research on different types of reviews has contributed to the knowledge of how language works in various social contexts where giving a personal opinion and an evaluation is the main communicative purpose. Under the review genre, academic reviews such as book reviews, review articles and blurbs have been the foci of linguistic research for many years. Reviewing the work of others not only offers valuable insights into the disciplinary communities, but investigating the characteristics of the genre has also deepened our understanding of the construction of evaluation and opinions in academic settings (e.g. Motta-Roth, 1995; Hyland and Diani, 20o9; Salmani Nodoushan and Montazeran, 2012). However, recent technological development and popularisation of online reviews have given rise to a host of research on this emerging digital genre, which includes online reviews of books (e.g. Mackiewicz, 2008), movies (e.g. Taboada, 2011), and products and services (e.g. Skalicky, 2013; Vásquez, 2014). This trend has expanded the scope of research on reviews from the expert evaluation of specialised fields to the evaluation of everyday products and services by members of the general public.

\subsection{From Traditional Academic Reviews to Online Customer Reviews}

Traditional book reviews focus on the expert reviews of academic books and articles in areas that are highly specialised such as psychology, neuroscience, 
linguistics, etc. The audience includes members of the scholarly community who share the same interest or discipline. Research on academic reviews can be divided into two general types: the first type is focused on the generic structural organisation of book reviews and the second type is concerned with the linguistic construction of evaluation and the writer-reader relationship. Motta-Roth (1995) conducted a genre analysis of book reviews on three different disciplines: linguistics, chemistry and economics. The findings revealed that academic book reviews can be broadly categorised into four generic stages: Introducing the book ${ }^{\wedge}$ Outlining the book ${ }^{\wedge}$ Highlighting parts of the book ${ }^{\wedge}$ Providing a closing evaluation of the book (where the caret symbol $\wedge^{\wedge}$ indicates relative order). In a genre study of school-based book reviews conducted by Martin and Rose (2008), a similar generic structure is identified although with different headings: Context of the story ${ }^{\wedge}$ Description $\wedge$ Judgment. Research on academic reviews shows that evaluative features play an important role in creating a writer-reader dialogue. Writers of academic reviews use praise skilfully and take considerable care to soften criticism through the use of hedges to mitigate the review, and to align values and display an understanding of the discourse community (Hyland and Diani, 2009).

Recent technological development on the Internet and social media has lent itself to the popularisation of online reviews that are written by consumers for consumers. Unlike academic reviews that are written by scholars or experts in the specific field on highly specialised topics, online reviews are written by the users or consumers of products or services. Review websites and dedicated review sections of product and service websites have made it possible for users or consumers to publicly share their personal opinions and judgments of the products or services. This new type of public discourse has given rise to the digital genre of online review, which has also attracted the attention of linguists and discourse analysts. From the study of the generic stages of movie reviews (Taboada, 2011), to the rhetorical focus of product reviews (Skalicky, 2013) and the lexical features in restaurant reviews across languages (Hong et al., 2016), findings from online reviews have offered a variety of insights and perspectives to enrich our understanding of the emerging digital genre. Virtanen (2017), in the context of studying online reviews of linguistics textbooks, proposes that the different types of online review genres (whether professional or for the general public) lead to the creation of discourse communities (Swales, 1990). These online discourse communities share communicative purposes, audience design and common style and text-structuring patterns. It is these emerging text-structuring patterns that we focus on in our analysis of generic structure in this paper.

The investigation of the generic structure and linguistic features that constitute evaluation and attitude has become the centre of research focus in the 
online review genre. In the case of online movie reviews, two broad generic stages (Description and Evaluation) were identified with corresponding lexicogrammatical properties that distinguished one from the other (Taboada, 2011). It is found that Evaluation contains more evaluative words than Description and is more frequently marked by causative connectives, whereas Description is characterised by temporal markers. Similarly, these two broad stages were identified in a comparative study of online reviews given by consumer and professional critics, which revealed that consumer critics tend to 'evaluate' more from a subjective perspective, while professional critics are more inclined to 'describe' by placing the movie in context (De Jong and Burgers, 2013). Using generic moves and rhetorical patterns to characterise online product reviews, Skalicky (2013) identified nine main categories of moves in which 'evaluation' is a prominent component. In addition, the study shows that reviews that are considered to be 'helpful' are those that provide experience-based information, as compared to new-information based or search-based with a rhetorical focus on the author or the product, but not the reader.

Apart from characterising the online review genre from the perspective of generic structure and stages, opinion mining and sentiment analysis are also commonly used methods for investigating the linguistic features of evaluation. These computational linguistic studies perform the automatic extraction of words and phrases that convey opinion and attitude in reviews with the goal of predicting the sentiment orientation (i.e. positive, negative or neutral) of the reviewed item (cf. Turney, 2002; Thet et al., 2010). However, the results are limited to word and sentence level. Other studies expand the investigation to include both the semantic and lexical levels by examining the discourse relations (relations between propositions) in the interpretation of evaluation and opinion words (e.g. Taboada and Grieve, 2004; Trnavac and Taboada, 2012; Trnavac et al., 2016). These studies combined Rhetorical Structure Theory (RST) and Systemic Functional Linguistics (SFL) to provide a more fine-grained analysis with richer interpretation of the texts. For example, Trnavac and Taboada (2012) found that concessive relations have the effect of reversing the polarity of evaluative words while condition relations contribute to the intensification or downtoning of an evaluation. Moreover, Trnavac et al. (2016) found that most opinion words are positioned in the nucleus of a discourse relation, suggesting that nuclei can be the focus for the automatic extraction of evaluation.

\subsection{Contrastive Analysis of Different Kinds of Reviews}

Over the past decade, the review genre has also attracted research interest from a cross-linguistic perspective, particularly the variation in the construction of evaluation across different languages. From a contrastive pragmatic perspective, the expression of evaluation can be measured in terms of degrees of 
subjectivity, orientation (whether toward the addressee or content), directness or indirectness in compliments and criticisms (cf. Kranich, 2016: Chapter 6) or degrees of impoliteness (Feng and Ren, 2020). Cross-culturally, it is observed that Spanish writers of academic book reviews tend to use less negative evaluation and are less critical than their American counterparts (Moreno and Suárez, 2008). Similarly, Japanese book reviews are found to contain fewer compliments when compared with English reviews, and Japanese reviewers tend to write about their personal feelings and their expectations of the book, instead of complimenting the authors or editors directly as in English book reviews (Fujimura-Wilson, 2017). Feng and Ren (2020) found that online consumer reviews written in English contain more positive impoliteness than their equivalent reviews in Chinese, whereas the opposite is true for negative impoliteness. In other words, English reviews feature more threats to positive face, whereas Chinese reviews tend to threaten negative face (see Brown and Levinson, 1987 for the concepts of positive and negative face in politeness research).

Recently, online reviews have also been used to investigate cross-cultural differences, especially in the relationship between broad cultural values such as 'individualism' and 'collectivism' and the review characteristics. For example, reviewers from 'collectivist' cultures are less likely to write reviews with low ratings (Fang et al., 2013; Fujimura-Wilson, 2017), whereas those from 'individualist' cultures are more likely to write reviews that enable self-expression (Chung and Darke, 2006). However, contrastive analysis of this kind is subject to issues such as predetermined criteria that are largely based on set values from previous literature, e.g., the preconception that Japanese and Chinese speakers are generally less assertive and more prone to conformity than Americans, and that the measurement of emotions is only based on words (Hong et al., 2016). Approaching from the perspective of speech acts with a focus on the construction of evaluation across English, Dutch and Italian online hotel reviews, Cenni and Goethals (2017) found that the three language groups adopt relatively similar up-scaling and down-scaling strategies in their evaluative statements. However, negative reviews written in English tend to include more positive comments than in Italian and Dutch, which could be explained by the general face-saving politeness strategies that are culture-specific.

\subsection{SFL Approach to Contrastive Analysis}

With the aim of investigating the cross-linguistic variation of language use in context, some linguists have adopted Systemic Functional Linguistics (SFL) in the contrastive analysis of different genres. With a fully integrated platform 
that consists of analytical tools linking context to semantics and lexicogrammar, the SFL framework enables language comparisons at a different scale and scope, from linguistic typology (Matthiessen et al., 2008) to translation studies (Matthiessen, 2001; Steiner and Yallop, 2001; Steiner, 2004). For example, Taboada et al. (2014) examined online movie reviews in English, German and Spanish at the semantic level. Using the subcategories of Attitude and Graduation under the Appraisal framework (Martin and White, 2005), the study revealed a number of cross-linguistic variations, which could be attributed to lexicogrammar, word order, argumentative style and sociocultural reasons. In a cross-linguistic analysis of online book reviews in English, Japanese and Chinese, Chik (2018) observed that although the generic structure is similar across the three languages, the selection of Theme (textual/ logical, interpersonal, circumstantial and topical) is very different: English reviews mainly unfold through textual/logical Themes, Japanese through circumstantial Themes and Chinese through a combination of textual/ logical, interpersonal and circumstantial Themes. In this study, we propose an integrated methodology that incorporates the SFL and RST frameworks to investigate the characteristics of online book reviews, with the aim of identifying the cross-linguistic variation across semantic and lexicogrammatical levels between English, Japanese and Chinese (cf. Chik, 2017). In the next section, we will discuss the methodology that we have undertaken for this study.

\section{Methodology}

\subsection{Data}

The corpus consists of 60 book reviews that were posted on the Customer Reviews section of the Amazon website between 2010 and 2018. All reviews concern the book titled "1Q84", a novel written by Japanese writer Haruki Murakami. The novel was written in Japanese and translated into other languages, including English and Chinese. The data comprises equal numbers of positive and critical reviews in the different Amazon websites that serve the respective language communities. We randomly selected 20 reviews in each language from the first 10 pages of each positive and critical review category.

In general, there are more positive reviews of this book (English $-66 \%$, Japanese $-61 \%$ and Chinese $-90 \%$ ) than critical reviews (English $-34 \%$, Japanese $-39 \%$ and Chinese $-10 \%$ ). Positive reviews were usually given 4 to 5 stars out of a 5 -star rating system while critical reviews ranged from 1 to 3 stars in the reviewer's opinion. The star ratings are calculated based on Amazon's 
machine learning model that takes into account factors including the age of a rating, whether the ratings are from verified purchasers, and factors that establish reviewer trustworthiness.

\subsection{Analytical Frameworks}

We employ two attested frameworks to analyse the structural construction of online book reviews. The first is the genre approach (Martin, 1984), which describes the generic structure of the text in terms of the generic elements or stages that characterise the genre under study. The generic stages are functional in nature and are determined by the genre's communicative purposes. For example, Martin and Rose (2008: 93-94) identified three generic stages in academic book reviews: Description, Evaluation and Judgment. In addition to the generic stages that constitute the online book review genre, we are also interested in the cross-linguistic variation, if any, between English, Japanese and Chinese. The reviews are analysed and compared across the three languages in terms of frequency, sequence order (beginning, middle and end) and the obligatory/optional status of the generic stages.

To analyse the rhetorical structure of online book reviews, we use Rhetorical Structure Theory or RST (Mann and Thompson, 1988), a framework for describing the rhetorical relations between parts of a text. According to RST, texts are organised into functional units that enter into different types of rhetorical relations in a recursive manner. Relations are defined to hold between two text spans or elementary discourse units (EDU), which mostly carry different 'weight', that is, one unit is more central to the writer and the other one serves a supportive function. The EDU that plays the central role is called the nucleus while the supportive unit is the satellite. As illustrated in Example (1), the nucleus is linked to the satellite through a Cause relation signalled by the connective because.

(1) Nucleus-Satellite relation: Cause

$\mathrm{N}$ : I quickly purchased it

$\mathrm{S}$ : because Mr. Murakami is one of my faves.

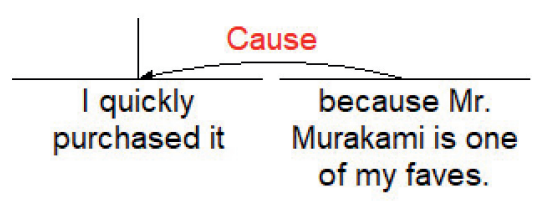


Moreover, relations are sometimes defined to hold between two or more EDUs that are of equal 'weight' in a multi-nuclear relation, as in Example (2), where the first EDU is in a Contrast relation with the second EDU.

(2) Multi-Nuclear relation: Contrast

$\mathrm{N}$ : Don't let the length of this novel scare you off.

$\mathrm{N}$ : Plunge in soon!

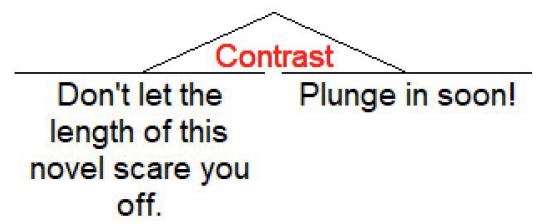

The set of relations available for RST analysis is, in principle, open and can be modified according to the type of text under analysis. There are about 20 relations, which connect different spans of text based on the degree of importance as well as hierarchy with respect to its related spans. Table 1 presents a list of different rhetorical relations that were employed during the RST analysis in this study.

TABLE 1 Different types of rhetorical relations

\section{Nucleus-Satellite Relations}

\begin{tabular}{lll}
\hline Background & Otherwise & Evidence \\
Preparation & Condition & Evaluation \\
Circumstance & Cause & Solutionhood \\
Elaboration & Purpose & Restatement \\
Concession & Result & Motivation \\
Antithesis & Justify & Enablement \\
\hline
\end{tabular}

Multi-Nuclear Relations

$\begin{array}{lll}\text { Contrast Joint Sequence } & \text { J }\end{array}$




\subsection{Annotation and Analytical Procedures}

We annotated all 6o online book reviews by coding the generic stages based on the communicative purposes of the genre. The coding scheme was derived from the categories identified in the 60 reviews and was applied to the three language versions in the corpus. Taking into consideration the generic stages proposed in other review genres in the literature, we identified the high-level categories of online book reviews and refined the sub-categories based on the texts that we analysed. Starting from the broad stages of academic book reviews (Motta-Roth, 1995), we identified the generic stages related to the background, introduction and evaluation of the book. We then further finetuned the evaluation stage into different sub-categories, including Plot and Characters as in movie reviews (cf. Taboada, 2011). Furthermore, we grouped the background, introduction and general comment stages into a generic stage called Metapragmatic Comment, a term borrowed from the genre of hotel reviews (Cenni and Goethals, 2017). Finally, we identified the sub-categories of the recommendation stage based on a similar rhetorical focus as that identified in product reviews, that is, either author-focused or reader-focused (cf. Skalicky, 2013). Table 2 presents the three broad generic stages, with their corresponding sub-categories, that were identified for this study.

Metapragmatic Comment (MP) is a stage that contains comments that serve as a preparative and mitigating sentence for the comments that follow. Commonly found in hotel reviews (cf. Cenni and Goethals, 2017), these comments are generally used to profile the reviewers e.g. as 'non-complainers' in negative reviews, and as instances of meta discourse. Evaluation (EV) is a broad stage and can be further categorised into evaluation of the book overall (EV:BK), author (EV:AU), plot (EV:PL) and character (EV:CH). At the recommendation stage (RC), the recommendation can be directed towards the reader (RC:RD) or author (RC:AU).

In terms of lexicogrammatical analysis, we focus on features that realise the main communicative functions of online book reviews, namely evaluation and recommendation. Results from previous studies on different review genres show that evaluative comments are mainly realised in individual words and phrases (cf. Taboada, 2011; Tsujii et al., 2017). In our study, we examine nouns, verbs, adjectives and adverbs that are evaluative and try to identify any salient patterns associated with the different stages. Below are some examples of evaluative words from our corpus.

Nouns: masterpiece, poor work, gimmick

Verbs: lacked, waste, fails

Adjectives: enjoyable, amazing, painful, boring 
TABLE 2 Proposed generic stages of online book reviews

Generic Stages

Description

\section{Primarycategory Sub-category}

Metapragmatic -

Comment (MP)

Evaluation (EV)

Book overall (EV:BK)

Author (EV:AU)

Plot (EV:PL)

Character (EV:CH)

Recommendation Reader (RC:RD)

(RC)

Author (RC:AU)

comments that serve to prepare the

reader for the evaluation that follows and are generally used to profile the reviewers and as instances of meta discourse

overall evaluation of the book

evaluation of the author (or translator in

English and Chinese versions)

evaluation of the plot, structure and flow of the story

evaluation of the characters in the novel recommendation directed towards the reader

recommendation directed towards the author, and sometimes to the editor or translator

For features that constitute recommendation, we analyse lexicogrammatical resources that are selected to realise the speech act of recommending, which can be lexical e.g. recommend, suggest and grammatical e.g. in the form of mood (imperative mood -Don't buy) and modality (e.g.you can read Norwegian Wood instead). To measure the degree of modal responsibility assigned to the reader or addressee, we categorise the speech act of recommending along the cline of modality as follows (from highest degree to lowest degree): Read it! - You must read it. - You should read it. - I recommend that you read it. - You can read it. (cf. Matthiessen et al., 2008: 198)

To identify discourse markers that signal the rhetorical relations between EDUs, we examine the different conjunctions that organise the logical development of the discourse (cf. Matthiessen and Teruya, 2015). For example, a concession relation can be signalled by a causative conjunction such as although and while, and a circumstance relation can be signalled by a temporal conjunction such as when and after.

To facilitate the annotation of rhetorical relations, we used rstWeb (version 2.0.3), a web-based annotational tool for RST analysis. Six sample 
texts ( 3 positive and 3 critical reviews) from each language in the corpus were uploaded to rstWeb for text analysis. rstWeb was selected over other RST analytical tools, such as UAM, because it is easy to use and supports both iOS and Android as well as all three languages being studied. In addition, it provides the tools for EDU segmentation as well as the tagging of rhetorical relations, while allowing the specification of the hierarchical structure of the entire text. The result is a visualisation of the text in a RST tree (see Figure 1 below).

There are two parts to the annotation procedure. The first part is concerned with the annotation of the generic stages and is performed over all 60 texts in the corpus. The tasks are summarised below.

- Identify the generic stages of each review by classifying the stages (e.g. MP, $\mathrm{EV}, \mathrm{RC}$ ) based on the communicative purposes of the online book reviews.

- Examine the frequency of occurrence of each stage, the sequence order and whether the stages are obligatory or optional.

- Identify salient lexicogrammatical features that characterise the different stages.

- Compare the similarities and differences across the three languages.

The second part covers the annotation of rhetorical relations using rstWeb. Since RST analysis is a detailed manual process that requires cross-checking and verification between the annotators, we chose only a selection of texts, and not the entire corpus, for this step. The annotation is performed over 18 texts (6 from each language) and consists of the following tasks.

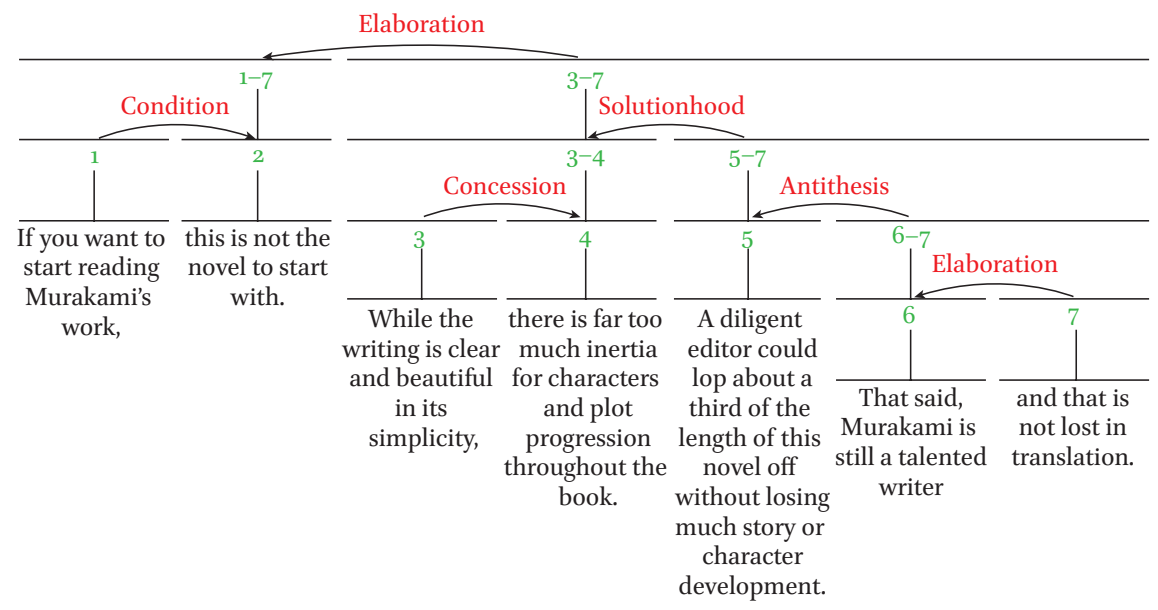

FIGURE 1 Example of the RST analysis of an English online book review (Text 15) 
- Segment the text into EDUs, primarily at the clause level, but could include phrases or multiple clauses that serve as a functional unit

- Decide on the nuclearity (nucleus-satellite or multi-nuclear relations) and the type of rhetorical relation to be applied between the EDUs

- Connect the EDUs by tagging the units with selected rhetorical relations

- Specify the hierarchical structure of the text

The completed RST tree is then mapped against the generic stages that have been identified in part one of the annotation process to present a visual representation of the entire text. Finally, we describe the rhetorical patterns in relation to the corresponding generic stages from a cross-linguistic perspective.

\section{$4 \quad$ Results and Discussion}

\subsection{Generic Stages in Online Book Reviews}

Our analysis of online book reviews reveals three broad stages that constitute the genre: Metapragmatic Comment, Evaluation and Recommendation. Of the three generic stages, only Evaluation is obligatory (cf. online movie reviews in Taboada, 2011). This pattern is shared between the three languages under examination and there is no observable difference between positive and critical reviews. Evaluation can be further sub-categorised into evaluation of the 'book overall' (EV:BK), 'author' (EV:AU), 'plot' (EV:PL) and 'character' (EV:CH), while Recommendation can be sub-divided into recommendation for 'readers' (RC:RD) and 'author' (RC:AU).

In terms of frequency of occurrence, Evaluation is the most frequent stage in online book reviews $(76 \%)$, followed by Metapragmatic Comment $(14 \%)$ and Recommendation (10\%). This result is not surprising given that the goal of reviews is to evaluate a product or service, in this case a novel that has been purchased on Amazon. Within the broad category of Evaluation, the most frequent sub-type is 'book overall' (EV:BK) and this finding is consistent across all three languages. However, it is interesting to note that English and Chinese reviews share the same preference for the other evaluation sub-types in descending order of frequency (EV:AU $>\mathrm{EV}: \mathrm{PL}>\mathrm{EV}: \mathrm{CH})$, while Japanese reviews display a preference for the reverse order (EV:CH $>\mathrm{EV}: \mathrm{PL}>\mathrm{EV}: \mathrm{AU})$, as presented in Table 3.

In cases where all three generic stages are present, the preferred order is Metapragmatic Comment ${ }^{\wedge}$ Evaluation ${ }^{\wedge}$ Recommendation, which is common in English and Chinese reviews. The general tendency is to begin the review with some metapragmatic comments, which serve to prepare the reader for the evaluative comments that follow, while recommendations are generally given at the final stage. In the recommendation stage, the reviewer concludes 
TABLE 3 Frequency distribution of the generic stages in English, Japanese and Chinese online book reviews

\begin{tabular}{|c|c|c|c|c|c|c|c|}
\hline & \multirow{2}{*}{$\begin{array}{l}\text { MP } \\
(14 \%)\end{array}$} & \multicolumn{4}{|c|}{$\operatorname{EV}(76 \%)$} & \multicolumn{2}{|c|}{ RC (10\%) } \\
\hline & & EV:BK & EV:AU & EV:PL & EV:CH & RC:RD & RC:AU \\
\hline English & 10 & 26 & 17 & 11 & 9 & 11 & 4 \\
\hline Japanese & 9 & 27 & 7 & 8 & 13 & 0 & O \\
\hline Chinese & 10 & 19 & 9 & 6 & o & 5 & o \\
\hline Total & 29 & 72 & 33 & 25 & 22 & 16 & 4 \\
\hline
\end{tabular}

Note: MP - Metapragmatic Comment

EV:BK - Evaluation: Book Overall

EV:AU - Evaluation: Author

EV:PL - Evaluation: Plot

EV:CH - Evaluation: Character

RC:RD - Recommendation: Reader

RC:AU - Recommendation: Author

$n=201$ (total number of occurrences of each generic stage type in the corpus -60 texts in total and or 20 per language)

the review by either 'recommending' or 'not recommending' the book to the reader.

(3) English positive review (Text 2)

Generic stages:

Metapragmatic Comment $\wedge$ Evaluation (book overall $\wedge$ author $\wedge$ character ${ }^{\wedge}$ plot $)^{\wedge}$ Recommendation (reader)

\section{0 out of 5 stars A pleasure to read}

[MP] When one reads a book by Murakami it is not about the plot or finding out what happens at the end; it is about the reading experience. [EV:BK] Reading a book by Murakami is simply enjoyable to read. [EV:AU] He is a master of drawing the reader into the story, revealing more details as the story progresses. It is similar to how we get to know people in real life, we learn about others best by sharing experiences with them. [EV:CH] One significant character (the dowager), we don't even learn her actual name until close to the end of the book. By the end of the book I felt like Tengo and Aomame (even Ushikawa) were friends, not just characters. I was a little sad when the book ended and I had to leave them. 
[EV:PL] Having said that, the book does have plots and action and suspense. It is at once a romance, a detective story, a family drama, a fantasy, a thriller. It is, in fact, one of the most unusual romances ever. It makes social/political/religious commentary, although Murakami is so good at presenting multiple points of view, I could not tell his personal view, except he obviously thinks there is no point in an ordinary life.

[RC:RD] But, don't read it for those reasons. Read it, because it is just plain enjoyable.

However, it is worth noting that the recommendation stage tends to be more popular in English reviews and is conspicuously absent in Japanese reviews, in which case the preferred order is Metapragmatic Comment ${ }^{\wedge}$ Evaluation. Also, in Chinese reviews, the recommendation stage does not occur as frequently as in its English counterpart, and the recommendation is directed mainly towards the reader rather than the author e.g. [RC:RD] 建议只借不买 “I recommend to borrow and not to buy" as in Example (16).

\subsection{Rhetorical Patterns at the Global Level}

In this section, we provide a description of the rhetorical patterns that characterise online book reviews with reference to the generic stages identified. In addition, we examine the salient lexicogrammatical features that realise these patterns from a contrastive perspective. Finally, we discuss how a crosslinguistic analysis that takes into consideration the generic structure, rhetorical patterns and lexicogrammatical features can provide a rich source of analyses for the study of contrastive pragmatics.

The results of the RST analysis show that the rhetorical relations identified in the three languages under examination constitute some recurrent and relatively similar patterns. First of all, in terms of the frequency of occurrence, Elaboration, which represents about one-third of all the relations, is the most frequently selected type of relation across all the languages (English $32 \%$ / Japanese $38 \%$ / Chinese $33 \%$ ). This general relation is selected both at the global and local levels to link satellites that contain details, examples and explanations to support the nucleus. This result is consistent with other work in RST that shows that Elaboration is a frequently occurring relation (Carlson et al., 2001; Taboada, 2004; Zeldes, 2019), in part due to its polyfunctionality as a relation among propositions, but also among entities in the text (Knott et al., 2001). 
TABLE 4 Frequency distribution of the rhetorical relations in English, Japanese and Chinese online book reviews

\begin{tabular}{|c|c|c|c|c|c|c|}
\hline \multirow{2}{*}{$\begin{array}{l}\text { Rhetorical Relations } \\
\text { Background }\end{array}$} & \multicolumn{2}{|c|}{ English } & \multicolumn{2}{|c|}{ Japanese } & \multicolumn{2}{|c|}{ Chinese } \\
\hline & 2 & $2 \%$ & 2 & $3 \%$ & 2 & $2 \%$ \\
\hline Preparation & 4 & $5 \%$ & 1 & $1 \%$ & 3 & $3 \%$ \\
\hline Circumstance & 5 & $6 \%$ & 6 & $8 \%$ & 7 & $7 \%$ \\
\hline Elaboration & 28 & $32 \%$ & 30 & $38 \%$ & 31 & $33 \%$ \\
\hline Concession & 10 & $11 \%$ & 7 & $9 \%$ & 4 & $4 \%$ \\
\hline Antithesis & 3 & $3 \%$ & 10 & $13 \%$ & 1 & $1 \%$ \\
\hline Otherwise & o & ०\% & $\mathrm{o}$ & ०\% & $\mathrm{O}$ & ০\% \\
\hline Condition & 1 & $1 \%$ & o & ०\% & 1 & $1 \%$ \\
\hline Cause & 6 & $7 \%$ & 4 & $5 \%$ & 5 & $5 \%$ \\
\hline Purpose & $\mathrm{O}$ & ০\% & o & ०\% & o & ০\% \\
\hline Result & 1 & $1 \%$ & $\mathrm{o}$ & ०\% & 5 & $5 \%$ \\
\hline Justify & 2 & $2 \%$ & $\mathrm{o}$ & ०\% & 3 & $3 \%$ \\
\hline Evidence & 1 & $1 \%$ & 1 & $1 \%$ & o & ০\% \\
\hline Evaluation & $\mathrm{O}$ & ○\% & $\mathrm{o}$ & ०\% & $\mathrm{O}$ & ०\% \\
\hline Solutionhood & 2 & $2 \%$ & $\mathrm{o}$ & ०\% & o & ০\% \\
\hline Restatement & 1 & $1 \%$ & 3 & $4 \%$ & 1 & $1 \%$ \\
\hline Motivation & 2 & $2 \%$ & 1 & $1 \%$ & 5 & $5 \%$ \\
\hline Enablement & o & ०\% & $\mathrm{o}$ & ०\% & o & ০\% \\
\hline Contrast & 12 & $14 \%$ & 9 & $11 \%$ & 8 & $8 \%$ \\
\hline Joint & 5 & $6 \%$ & 6 & $8 \%$ & 19 & $20 \%$ \\
\hline Sequence & 2 & $2 \%$ & $\mathrm{o}$ & ०\% & o & ०\% \\
\hline
\end{tabular}

Other relations that are repeatedly selected include Circumstance, Concession, Antithesis, Cause, Contrast and Joint (see Table 4 for a breakdown of the distribution across reviews in the three languages).

Secondly, we observe that online book reviews tend to be rhetorically organised in a nucleus-satellite structure at the global level, which can be classified into two broad types. The first type, which is more common, consists of an evaluative comment that serves as the global nucleus. The entire review is then developed through the supporting global satellite that contains examples or details to substantiate the evaluative comment presented in the global nucleus.

(4) GN: Like others, I found this book extremely painful to finish.

Gs: It was boring, redundant, poorly edited, ...

Relation: Elaboration (Text 11) 


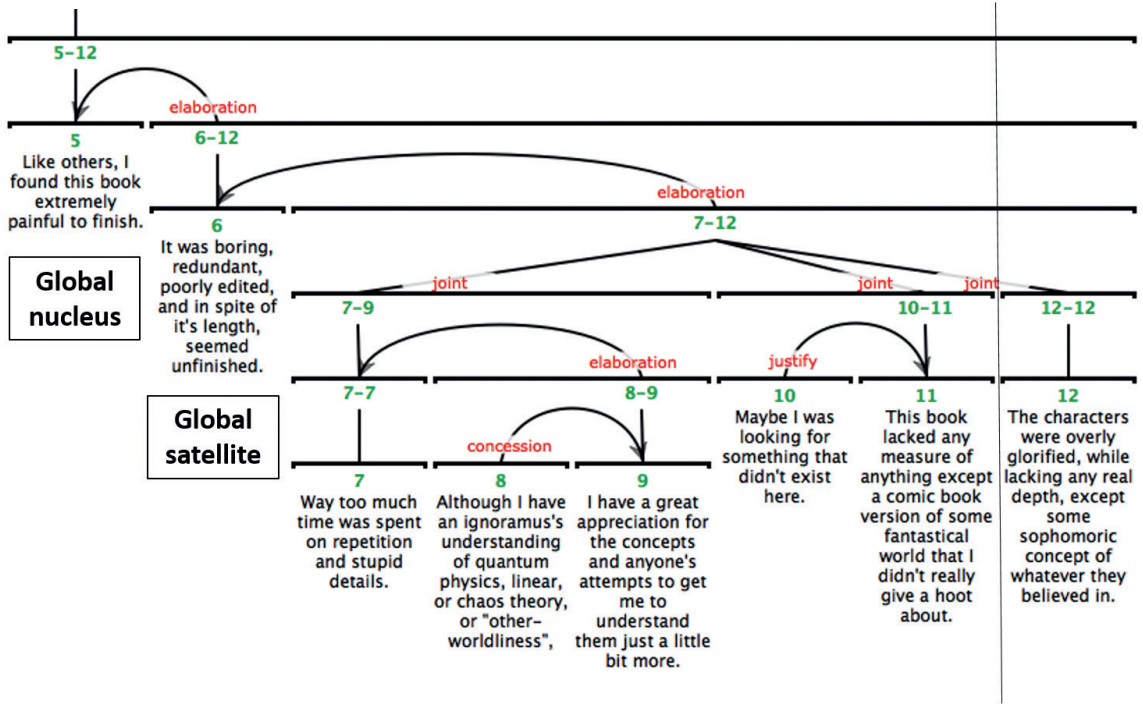

The review in Example (4) is built on the negative evaluation of the book overall - that it is 'extremely painful to finish'. The supporting satellites are organised in a list of examples and details linked together by several Joint relations, which function to elaborate the claims in the global satellite that it was boring, redundant, ...'

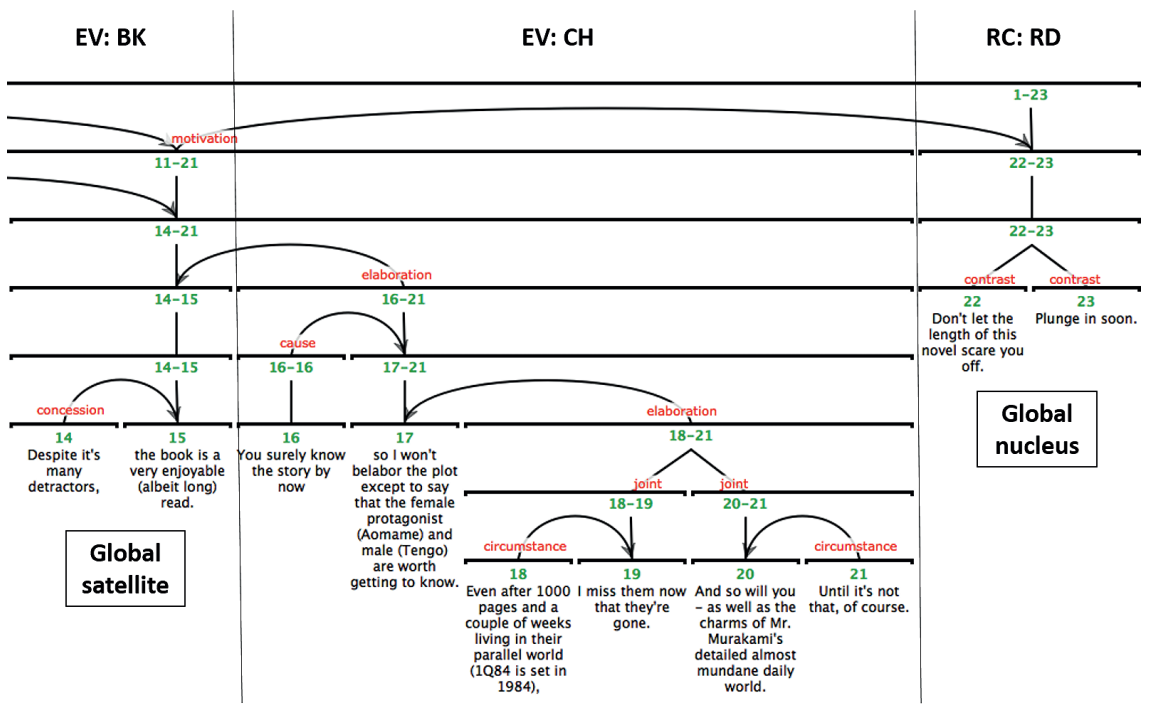


The second type of global rhetorical organisation centres on a proposed action for the reader. The reviewer makes use of the evaluative comments in the global satellite to fuel the recommendation in the global nucleus, which is usually a command or suggestion asking the reader to undertake a certain action.

(5) Gs: the book is a very enjoyable (albeit long) read GN: Don't let the length of this novel scare you off. Plunge in soon. Relation: Motivation (Text 5)

In Example (5), the review is set up for a call for action, which is linked to the positive evaluation presented in the global nucleus through a Motivation relation. The recommendation is built upon a series of evaluations that are substantiated with examples and reasons presented in the global satellite. The EDUs in the global satellite are linked by rhetorical relations such as Elaboration, Circumstance, Cause and Concession to increase the reader's inclination to accept the recommendation in the end.

Finally, we observe other typical relations that organise the EDUs at the global level of online book reviews, which include Preparation, Background and Restatement. While Preparation and Background usually connect EDUs within the metapragmatic comment stage or link the metapragmatic comment and evaluation stages together, Restatement connects the EDUs in the final stage, which serves to reinforce the evaluation or stance in the global nucleus that comes at an earlier stage, as illustrated in Example (6).

\begin{tabular}{|c|c|c|c|}
\hline 1 & 2 & & - \\
\hline$\downarrow$ & enternee & & ${ }_{\text {contrast }}^{\text {triti }}$ \\
\hline 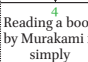 & $h^{5-16}$ & & 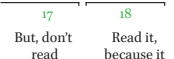 \\
\hline 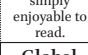 & l & elaboration & 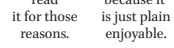 \\
\hline \begin{tabular}{|c} 
Global \\
nucleus
\end{tabular} & $\sqrt[5 \text { elaboration }]{5^{-10}}$ & $\begin{array}{l}\text { Haxing said } \\
\text { that hat }\end{array}$ & \\
\hline
\end{tabular}

(6) GN: Reading a book by Murakami is simply enjoyable. (EV:B K) $\mathrm{S}$ : Satellite: ... Read it, because it is just plain enjoyable. (RC:RD) Relation: Restatement (Text 2)

The Restatement relation at the final stage is usually signalled by lexical repetition or paraphrasing to reinforce the main evaluation of the review. In Example (6), the positive evaluation of the book as 'simply enjoyable' in the global nucleus is reframed as a reason, 'because it is just plain enjoyable', for the command to 'Read it' in the final recommendation stage. 


\subsection{Rhetorical Relations at the Local Level}

Rhetorical relations at the local level reflect the strategies employed by the reviewer to increase the reader's inclination to believe in the proposition, whether it is an evaluation or a recommendation. In the next section, we discuss how rhetorical relations are deployed to organise online book reviews into meaningful units to fulfil the communicative purposes in relation to the generic stages of Metapragmatic Comment, Evaluation and Recommendation.

\subsubsection{Metapragmatic Comment}

In the metapragmatic comment stage, the reviewer prepares the reader for the different stages of evaluation that follow by offering some background information about the review. We identified three general types of metapragmatic comments, which include 'self-profiling', 'backgrounding' and 'standardsetting'.

The 'self-profiling' type of metapragmatic comment typically consists of information that profiles the reviewer as an 'objective opinion giver' or as 'nonbiased' (Example 7a and 7 b), which adds to the credibility of the review. Common mitigation strategies include the use of a disclaimer to restrict comments to one's own experience and negation to contrast with the strong comments that follow (cf. Ren, 2018), whereas comments that serve as 'backgrounding', such as 'reason and circumstance' that lead to the purchase of the book, help set the scene for the evaluation to come (Example $7 \mathrm{c}$ and $7 \mathrm{~d}$ ). Finally, some reviewers make use of the metapragmatic comment stage to establish standards of what, in their opinion, constitutes a 'good read' or 'quality work'. This type of comment serves to set the stage for a comparison with the book under evaluation (Example $7 \mathrm{e}$ and $7 \mathrm{f}$ ).

a. I am not opposed to long books, but it seems fair that an author needs to earn the right to have his audience read 1,300 pages (Text 18)

b. 平时不爱读书也被深深吸引了呢。(Text 45)

"Normally I do not like to read books and yet I'm deeply mesmerised."

c. When 1Q84 came out a while ago, I quickly purchased it because Mr. Murakami is one of my faves. (Text 5)

d. 私は、海外に住んでいる者です....たまたま、こちらに住む日本人の方 が BOOK 1、2 を面白いから、ということで貸してくださいました。 (Text 24)

"I am someone who lives abroad ... and by chance, a Japanese friend of mine who lives here (in Japan) lent me the book ..." 
e. 良い作品の判断基準が、読み終えた後に、また読みたいと思うかどう かである。(Text 32)

"A good book is determined by whether you want to read it again right after you've finished reading it ..."

f. 喜欢林少华的译本, 淡淡的优美味 ... (Text 58)

"I like Lin Shao Hua's translation work, which has a subtlety in its beauty ..."

Overall, content in the metapragmatic comment stage functions to prepare and mitigate the comment that follows and can be said to be a characteristic of the online review genre (cf. Cenni and Goethals, 2017). The 'framing' placement of the self-referential disclaimers at the beginning of the review also strengthens the self-commodifying strategy in establishing solidarity with the online community (Virtanen, 2017). Rhetorical relations that typically occur in the metapragmatic comment stage include Preparation, Background, Concession, Antithesis and Circumstance. The RST diagram in Example (8) illustrates how the EDUs in the metapragmatic comment stage are rhetorically organised to support the main argument in the evaluation stage that follows.

(8) Text 18

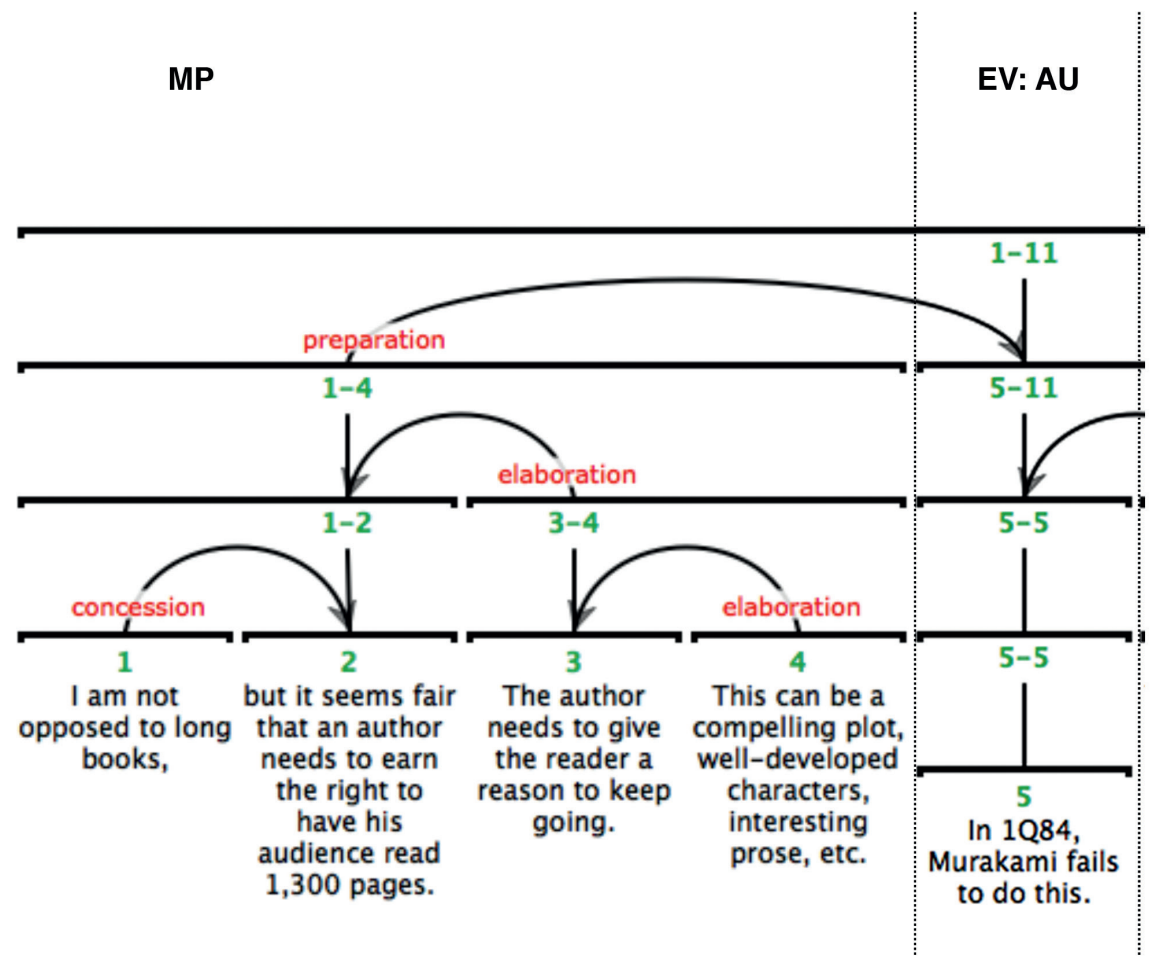


In Example (8), the metapragmatic comment serves two functions. The first function is to present the reviewer as someone who is not biased against long books, profiling him/her as an objective opinion giver. The second function is to establish a standard or requirement of what the reviewer considers to be a good piece of long work: 'a compelling plot, well-developed characters, interesting prose, etc.', all of which are elements that give the reader 'a reason to keep going'. The EDUs within the metapragmatic comment stage are organised primarily by Concession and Elaboration relations, while the nucleus of the metapragmatic comment ' ... an author needs to earn the right to have his audience read 1,300 pages ...' functions to support the central argument of the review, 'In 1Q84, Murakami fails to do this'. The metapragmatic comment prepares the reader for the negative evaluation that accuses the author of failing to give the reader a reason to read the book.

\subsubsection{Evaluation}

The RST analysis of our sample texts (six in each language) shows that online book reviews are globally organised in a nucleus-satellite structure where Evaluation is the centre of rhetorical development. Just as in movie reviews (cf. Taboada, 2011), Evaluation is the only obligatory stage in online book reviews and in most instances constitutes the global nucleus or the central proposition of the text. Among the different Evaluation sub-types, evaluation of the book overall (EV:BK) is most frequently selected as the global nucleus $(50 \%$ of English reviews, $67 \%$ of Chinese reviews and $83 \%$ of Japanese reviews), and is typically linked to the supporting satellites through an Elaboration or Evidence relation.

At the local level, other evaluation sub-types, such as evaluation of the author (EV:AU), plot (EV:PL) and character (EV:CH), form the main components of the overall schema, supporting the reviewer's propositions (evaluation of the book) and proposals (recommendations to the readers or the author). We observe some salient patterns in the selection of rhetorical relations that function to build evidence and reasons to support the central claim. Among those is the selection of Concession and Antithesis.

Antithesis relations tend to connect the nucleus to supporting satellites that present contrasting views or ideas to increase the reader's positive regard of the nucleus, which is favoured by the reviewer.

(9) English (Text 18)

Rhetorical relation: Antithesis

$S$ : The initial hook is incredible, and I was very excited to read. 
$\mathrm{N}$ : But by the $2 / 3$ mark I had lost significant interest. The prose became repetitive, which I suppose matched the plot.

(10) Japanese (Text 28)

Rhetorical relation: Antithesis

S: 「1284」は、他の長編作品より評価が低いようだが、

"Although it seems that $1 \mathrm{Q} 84$ has received lower ratings as compared to other long works"

$\mathrm{N}$ :そうは思わない。

"I don't agree."

In the English Example (9), the schema is characterised by a EDU that contains a contrasting nucleus-satellite pair. The satellite carries a positive evaluation of the book ('incredible' initial hook) while the nucleus contains a negative evaluation ('repetitive' prose), which is favoured by the reviewer. These contrasting experiences are linked by an Antithesis relation, which functions to increase the reader's inclination to believe in the negative evaluation in the nucleus.

Similarly, the satellite in the Japanese Example (10) presents a negative evaluation of the book by other reviewers ('lower ratings as compared to other long works'), which contrasts with the opinion of the reviewer in the nucleus ('I don't agree'). The nucleus is further elaborated with specific examples to increase the reader's positive regard towards the proposition of the reviewer, i.e. 1 Q84 is as good as, if not better than, the other long works by Murakami.

Concession relations, on the other hand, enable the reviewer to acknowledge a potential incompatibility between the situations presented in the nucleus and satellite.

\section{(11) English (Text 15)}

Rhetorical relation: Concession

$\mathrm{S}$ : While the writing is clear and beautiful in its simplicity,

$\mathrm{N}$ : there is far too much inertia for characters and plot progression throughout the book.

(12) Chinese (Text 50)

Rhetorical relation: Concession

$\mathrm{N}$ : 本来, 读者了解人物和环境已经很足够的程度,

"Both characters and settings are already good enough for the readers to comprehend,"

$S:$ 但是又多了那么些个多余的段落和细节。

"However, there are some unnecessary paragraphs and redundant details." 
As illustrated in Examples (11) and (12), the reviewer first affirms the positive points of the book ('characters and settings are already good enough') before presenting the negative evaluation ('unnecessary paragraphs and redundant details') through a Concession relation. By acknowledging the incompatibility of the opposing views, the reviewer is able to maintain a sense of objectivity in the evaluation.

Another prominent finding is the use of Contrast and Joint to expand the elaboration of the evaluation in the nucleus. Contrast and Joint relations in online book reviews serve to organise EDUs that are of equal weight, for comparing different parts of the book (Example 13) or listing different views or elements (Example 14) to exemplify or clarify the preceding evaluation.

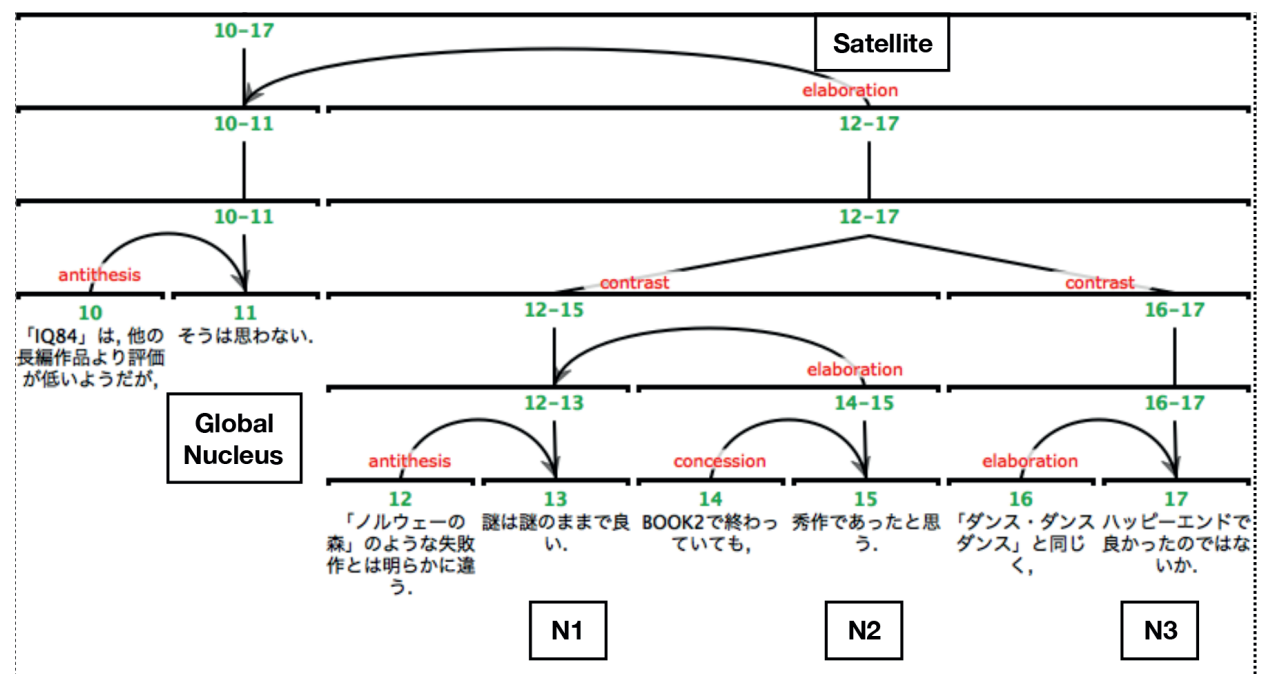

(13) Japanese (Text 28)

$\mathrm{N}$ : 「IQ84」は、他の長編作品より評価が低いようだが、そうは思わ ない。

"Although it seems that $1 \mathrm{Q} 84$ has a lower rating when compared with other long works by Murakami, I don't agree."

$\mathrm{S}\left[\mathrm{N}_{1}\right]:$ 「ノルウェーの森」のような失敗作とは明らかに違う。謎は謎 のままで良い。 ...

"It is good to remain mysterious, which is clearly different from previous failures such as 'Norwegian Woods."

$\mathrm{S}\left[\mathrm{N}_{2}\right]$ :「BOOK2で終わっていても、秀作であったと思う。

"Even if it ended with BOOK2, I think it's still a piece of great work." 
S [N3]:「ダンス・ダンスダンス」と同じく、ハッピーエンドで良かっ たのではないか。

"And isn't it great to have a happy ending just like 'Dance, Dance, Dance’?”

In (13), the global nucleus, which is a positive evaluation of the book, is presented as an opposing view to the negative evaluation it has received from other reviewers through an Antithesis relation. The claim is supported by comparing two other long works by Murakami, namely 'Norwegian Woods' and 'Dance, Dance, Dance'. The good and bad points of these two books are discussed in comparison with $1 \mathrm{Q} 84$, which collectively support the argument that $1 \mathrm{Q} 84$ should not be rated lower than other long works by Murakami. The rhetorical relation at work is Contrast, which is schematically one level below Elaboration, and this rhetorical relation organises the satellites to support the argument systematically.

Similarly, the Joint relation is frequently selected to elaborate the evaluation presented in the nucleus by listing examples and evidence with details that support the evaluation.

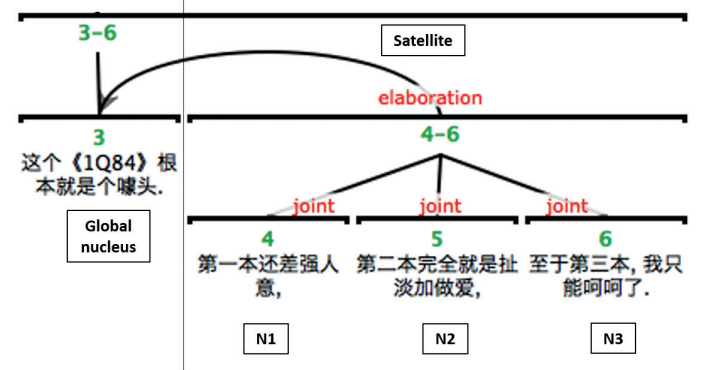

(14) Chinese (Text 55)

Rhetorical Relation: Joint

$\mathrm{N}$ : 这个《《1Q84》根本就是个噱头。

“This ${ }_{1 Q} 84$ is nothing but a gimmick."

$\mathrm{S}[\mathrm{N1}]$ : 第一本还差强人意,

"The first book is barely acceptable,"

$S[\mathrm{~N} 2]$ : 第二本完全就是扯淡加做爱,

"The second one is completely nonsense with added sex talk,"

$\mathrm{S}$ [N3]: 至于第三本, 我只能呵呵了

"As for the third book, I can only laugh out loud ..." 
In Example (14), the review is developed around the nucleus, which is a negative evaluation of the book accusing it of being just 'a gimmick'. Using a Joint relation to connect the specific evaluations of the three sections of the book, the reviewer presents the evaluation of each section in an equally weighted manner. In our corpus, the Joint relation is found to be favoured by Chinese online reviews $(20 \%)$, although similar rhetorical patterns are also observed in English and Japanese reviews but with lower frequency (6\% for English and $8 \%$ for Japanese).

\subsubsection{Recommendation}

Our study shows that Recommendation is an important component of the online book review genre, although it is not an obligatory stage. In our corpus, $50 \%$ of English reviews and $20 \%$ of Chinese reviews contain some form of recommendation, most of which are presented towards the end of the text. It is interesting to note that in English reviews, there are an equal number of recommendations in positive and critical reviews, while in Chinese reviews, there are more recommendations in critical reviews. Moreover, in English reviews, recommendations to the author or editor are only observed in critical reviews, while positive reviews are all reader-oriented. In contrast, all the recommendations in Chinese reviews are directed toward the reader and none is author-oriented. In terms of rhetorical relations, the relation that typically characterises the recommendation stage is Motivation. The reviewer presents some evaluative comments about the book, which function as a motivation for the reader to act according to the recommendation that follows. In our corpus, most of the recommendations that are given toward the final stage of the review constitute a call for action, directing readers to perform some kind of action that is beneficial to them.

(15) English (Text 5)

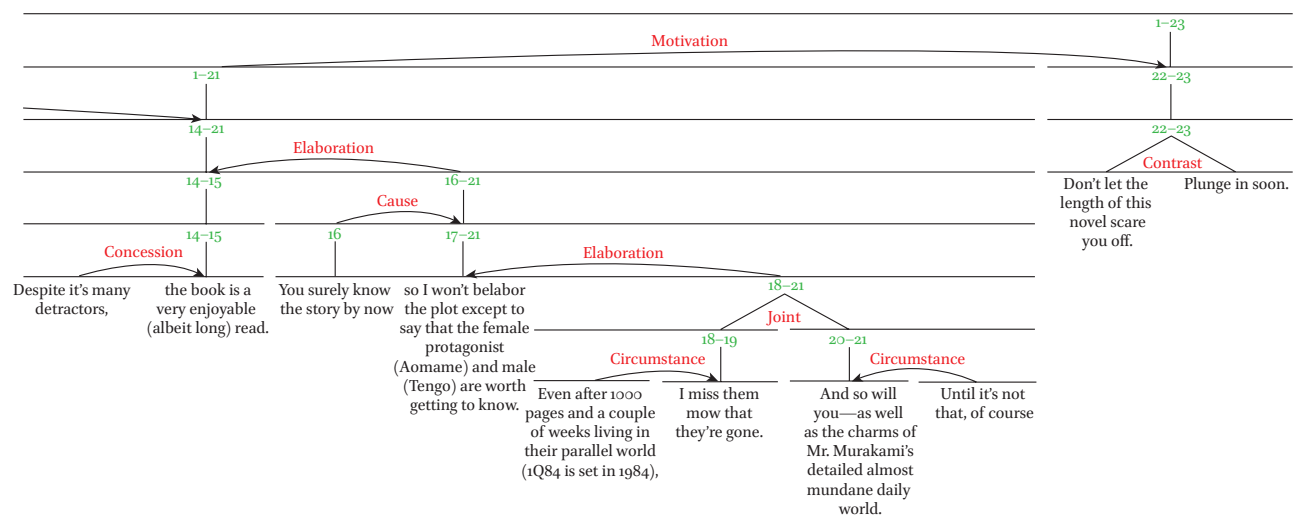


In Example (15), the entire review is built on a positive evaluation of the book: 'a very enjoyable (albeit long) read', which serves as the motivation for the recommended action 'Plunge in soon.' This type of direct and explicit recommendation, realised in an imperative mood, is common in English reviews, whereas in Chinese reviews, the preference is towards the use of expressions with explicit wording such as 'recommend' in a declarative mood e.g. '建议只 借不买 - I recommend to borrow and not to buy' (Example 16)

(16) Chinese (Text 58)

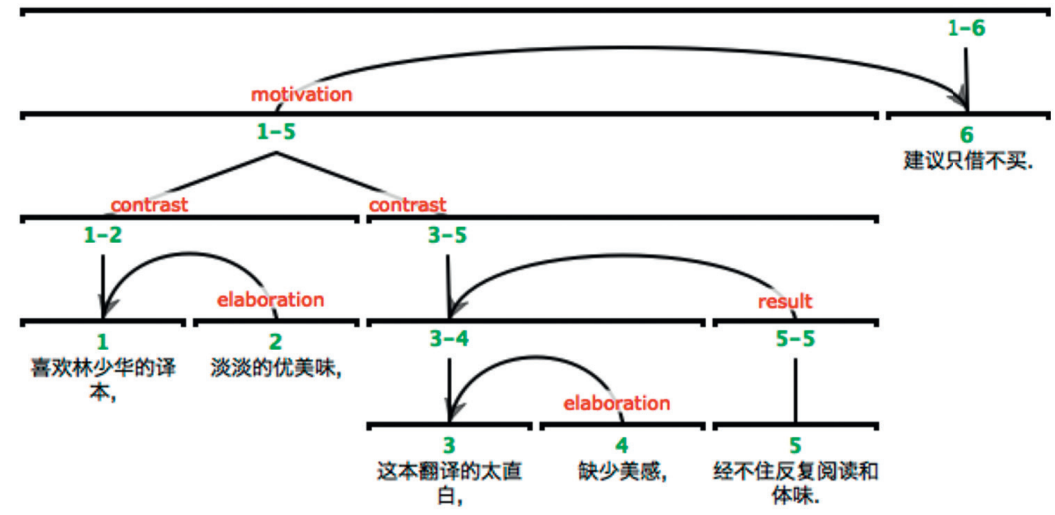

We also observe that a recommendation is sometimes given at the beginning of a review as a form of warning, especially in negative reviews. This pattern is parallel in both English (Example 17) and Chinese (Example 18) reviews, where the negative recommendation is supported by the satellite through a Condition relation.

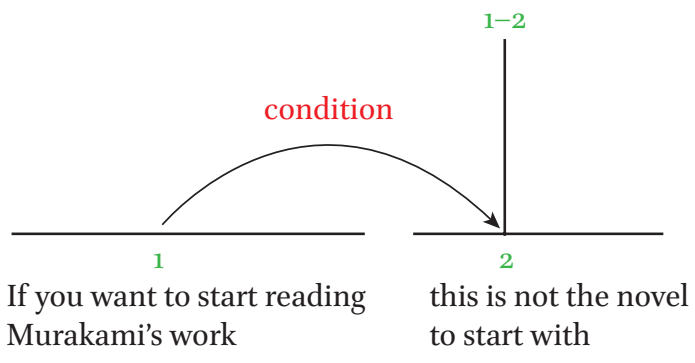

(17) English (Text 15)

[RC:RD]

S: If you want to start reading Murakami's work,

$\mathrm{N}$ : this is not the novel to start with. 


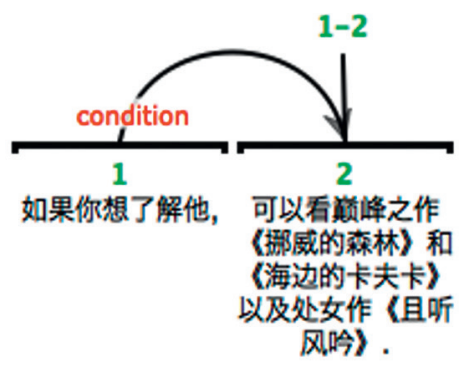

(18) Chinese (Text 55)

[RC:RD]

$S:$ 如果你想了解他,

"If you want to get to know him,"

$\mathrm{N}$ : 可以看巅峰之作《挪威的森林》和《海边的卡夫卡》以及处女作 《且听风吟》。

"you can read his peak works "Norwegian Wood" and "Kafka by the sea" and his debut work "Listen to the wind"."

It is interesting to note that the satellite is usually associated with the desire of the reader e.g. if you want in English or 如果你想 in Chinese, which functions as the condition for the recommendation. The Condition relation is usually signalled by the connective 'if' in English and 如果 in Chinese. This type of recommendation positions the reviewer as an expert in the author's work, indicating power and superiority over the reader. Instead of using direct commands, the reviewer adopts an indirect approach through the use of negative affirmative statements e.g. 'this is not the novel to start with', or those that are modulated e.g. 'you can read ...'. Whether the recommendation is an affirmative one or one that is suggestive in mood, the intention is to increase the reader's inclination to take the suggested action.

\subsection{Lexicogrammatical Features in Evaluation and Recommendation}

In terms of lexicogrammatical features, we examine lexical words such as nouns, adjectives, verbs and adverbs that are evaluative in nature, and compare the findings in English, Japanese and Chinese. We focus on the Evaluation and Recommendation stages because they contain the most evaluative content. We also investigate the different degrees of modality in the representation of recommendation in the three languages. Table 5 presents a sample selection of the lexicogrammatical features that are representative in constructing evaluation in the global nucleus and its supporting satellite. 
TABLE 5 Examples of the lexicogrammatical features that constitute evaluation in the global nucleus

$\begin{array}{ll}\text { Example } & \text { Global nucleus (Evaluation) } \\ & \text { Generic stage - EV:BK }\end{array}$
Lexicogrammatical

features that constitute

evaluation

Adjective + intensifier

+ adverbs: extremely

painful, boring,

redundant, poorly edited,

unfinished

Adjective:

決めつけのような

imposed

悪い

Bad

Adverb:兴奋的 excitingly

Adjective:

不错 pretty good

Adjective + intensifier:

很失望

extremely disappointing

$\mathrm{GN}$ : 但是内容却让我很失望。

But the content was extremely

disappointing.

(22) GN: But this book was like the most Metaphoric expression:

English soul-stirring elements and characters of like the most soul-stirring

Text 1 your elusive, fleeting dreams, come to life. Gs: It is worth trying ${ }_{1} \mathrm{Q} 84$ for the elements and characters possibility that it will leave you amazed of your elusive, fleeting and satisfied. dreams, come to life. Adverbial: worth trying Adjectives: amazed / satisfied 
TABLE 5 Examples of the lexicogrammatical features that constitute evaluation (cont.)

$\begin{array}{lll}\text { Example } & \text { Global nucleus (Evaluation) } & \text { Lexicogrammatical } \\ \text { Generic stage }- \text { EV:BK } & \text { features that constitute } \\ & \text { evaluation }\end{array}$

$\mathrm{GN}$ : 残念ながら、ノルウェーの森のよう

Japanese

に再読したい、と少しも思えなかった。

Text 32

(24)

Chinese

Text 50
Unfortunately, I did not have the slightest

desire to read it again like I did with

'Norwegian Wood'

GS: 結論から言えば、会話と話の流れが

二方的過ぎるのである。

In conclusion, the conversation and the

flow of the story are too unilateral.
Negation + expression of desire + comparison

再読したいと少しも思え なかった

did not have the slightest

desire to read it again

like 'Norwegian Wood'

intensifier:

一方的過ぎる

too unilateral

Adjective:

独特的 special

Metaphoric expression:

真的很值得细细品味

truly worth savouring.

$\mathrm{GN}$ : 真的很值得细细品味。

It's truly worth savouring.

We observe that the majority of the evaluation is associated with assigning values to the book or the experience of reading the book, which resonates with the findings of previous studies on evaluation and review genres (cf. Martin and White, 2005; Cenni and Goethals, 2017; Goddard et al., 2018). In our sample texts, evaluation is generally realised in adjectives, intensifiers, adverbs and metaphoric expressions. We observe a general trend across English, Japanese and Chinese in terms of the use of lexical words to construct evaluation in a direct manner. For example, in critical reviews, the book is assigned with negative values through the use of evaluative adjectives such as boring, redundant, unfinished (Example 19), or 很失望 'extremely disappointing' (Example 21). In other instances, the reading experience is attributed a negative value such as 読後感が悪い 'bad feeling after reading' (Example 20).

Another strategy is to indirectly attribute values or judgments to the book through the use of metaphoric expressions e.g. like the most soul-stirring elements and characters of your elusive, fleeting dreams, come to life in English and 真的很值得细细品味 'truly worth savouring' in Chinese. In Japanese, we 
observe a few instances that make use of negation, the expression of desire and comparison to construct an evaluation of the book. As illustrated in Example (23), the reviewer uses a comparison with Murakami's masterpiece 'Norwegian Wood' to indirectly bring about the negative evaluation of the book 1Q84 by negating the desire to read it again, 再読したいと少しも思えな かった 'I did not have the slightest desire to read it again', something that one would do if the book had been as great as Norwegian Wood, ノルウェーの森の ように 'like Norwegian Wood.'

While we do not observe any prominent divergence in the lexicogrammatical construction of evaluation across the three languages in our corpus, we detect some interesting patterns in the interpersonal grammar of recommendation. First of all, there is not a single case where the Japanese reviewer asks the reader to buy/not buy or to read/not read the book through the use of imperatives or explicit wordings such as 'I recommend/suggest' as in English and Chinese reviews. A closer examination of the data shows that Japanese reviewers prefer a more indirect way of conveying their recommended action. We investigated several instances in which a similar recommendation is given in English or Chinese and found that, in Japanese, instead of suggesting that the reader takes a particular action, the reviewer takes up the Actor role and the sentence is constructed as a future action that the reviewer will undertake (Example 25).

\begin{tabular}{lll} 
Language Recommendation & $\begin{array}{l}\text { Lexico- } \\
\text { grammatical features }\end{array}$ & $\begin{array}{l}\text { Degree of Modality } \\
\text { (obligation/ } \\
\text { inclination) }\end{array}$ \\
\hline
\end{tabular}

\begin{tabular}{lll}
\hline English & Try him! (Text 4) & imperative \\
Chinese & 建议只借不买 & Lexical 'recommend' \\
& “I recommend to borrow & speaker-oriented \\
and not to buy" (Text 58) & \\
Japanese & 私は、個人的に BOOK 4 & Lexical 'definitely buy' LOW \\
& が出てほしい! 絶対に & speaker-oriented \\
& 買います。 \\
& “As for me, I personally \\
& want book 4 to come out. \\
& I'll definitely buy it!” \\
& (Text 24) \\
\end{tabular}


The three instances in Example (25) represent different degrees of modality along the cline of obligation and inclination (Halliday and Matthiessen, 2014: 691-697). If we line up the three examples along this cline, we have, in order of declining degree of obligation/inclination: a command in English realised in an imperative mood 'Try him!' ^ a suggestion in Chinese realised in a modulated statement with an explicit expression of recommendation using lexical items such as 建议 'recommend' and 不买 'not to buy' ^ an expression of personal desire ほしい 'want' and a high degree of inclination to buy 絶 対に買います 'I'll definitely buy it!' in Japanese. Note that in the English and Chinese examples, the recommendation is directed toward the addressee, whereas in the Japanese example, although the degree of inclination toward the act of buying is high, the modal responsibility rests on the speaker. In our corpus, about half of the recommendations in English are construed as commands and the majority of the Chinese recommendations are expressed in statements with explicit wordings of 'suggest'. While this finding resonates with Matthiessen et al. (2008:196) that 'in Japanese, 'exhortative' commands realized by addressee-oriented 'imperative' clauses are uncommon", it will be interesting to investigate whether the selection of lexis, mood and modality in the recommending context is register dependent (see also Feng and Ren (2020) on the relatively low presence of on-record impoliteness in Chinese reviews). While it is beyond the scope of this study, we believe that a detailed investigation of the cross-linguistic analysis of lexicogrammatical features that construct evaluation in online book reviews will provide valuable insights into the contrastive pragmatics of this genre.

\subsection{Implications on the Dimensions of Contrastive Pragmatics}

We propose that the analysis illustrated in this paper can be adopted as an approach by which to explore pragmatics and discourse structure from a contrastive point of view. We have shown an analysis that works across all language strata, from the generic organisation through the rhetorical organisation and onto the lexicogrammar. Such an approach allows us to capture the overall characteristics of the genre under study in the three languages.

Our findings show that, at the global level, there is a tendency towards homogeneity, as illustrated by the similarity in rhetorical structure of online reviews across the three languages. However, at the local level, we observe some variation in language features that might have cross-cultural implications for the genre. For example, we find that both English and Chinese reviewers are more direct in giving recommendations in the form of orders using imperatives, while Japanese reviewers tend to indirectly suggest an action to the readers by discussing personal feelings and expectations (cf. Fujimura-Wilson, 2017). 
This could be attributed to the general cultural preference for indirectness in their communication strategy (Nakai, 2002), which is prominent in the speech act of giving a recommendation in the review context. Moreover, English reviewers tend to give advice to the authors or editors on how to improve their work, something that is not observed in Chinese and Japanese reviews. This deployment of positive impoliteness, which poses a threat to the positive face of the author, is also found to be a characteristic of English reviews (see the discussion on positive and implicated politeness between English and Chinese reviews in Feng and Ren (2020)).

It is also interesting to note that, regardless of whether the reviewers are evaluating the original Japanese version of the book or the translated English and Chinese versions, they tend to be either long-term fans of Haruki Murakami or first-time readers. While English and Chinese reviews may contain an evaluation of the author and/or the translator, and Japanese reviews only contain an evaluation of the author, if any, we do not observe any linguistic evidence that suggests a variation in language use for evaluation and recommendation that is influenced by reading the translated version of the book. However, it may be worthwhile investigating whether translation plays a role in influencing reviews of the same book in different languages.

The analysis can, of course, be expanded at each level. For instance, in conjunction with the genre analysis, one could undertake a more detailed register analysis of online book reviews as a genre, focusing on field, tenor and mode. One could examine thematic structures and their relationship to the rhetorical organisation of the text, in the form of textual progression. A comparison of processes and participants in the transitivity structure may reveal interesting contrasts in the three languages. Indeed, many possibilities present themselves. Our proposal is that the analysis across language strata that is enabled by SFL constitutes a fruitful avenue for contrastive pragmatics research.

\subsection{Limitations and Future Research}

The analysis we present in this paper can, of course, be improved and expanded upon. One limitation of the research is the relatively small sample size that was used for each of the languages. This is necessarily so, given the manual, intensive nature of both generic and RST analyses. In both cases, the analysis involves a close reading of the text, a consideration of the context in which it takes place and multiple rounds of analysis and revision. Nevertheless, a larger number of texts would allow us to ascertain whether the tendencies we have observed in our analysis are indeed tendencies or perhaps artefacts of the texts we collected. This would not make the analysis any less valid, as the type of 
discourse analysis we propose here is worthwhile for each text in isolation, regardless of whether it extends to other instances of the same genre.

Potential future avenues of research could expand on each of the areas we have analysed. In the generic structure, one could undertake a more in-depth analysis of the register variables (field, tenor and mode) and a comparison with cognate genres, online and offline, for each of the languages. In the RST analysis, a particularly interesting area of research is the signalling of rhetorical relations with conjunctions, discourse markers or through other means (see Das and Taboada (2018) for a discussion of signalling in RST). Finally, the study of the lexicogrammatical properties of the texts could be further expanded, to include other categories, and to more formally relate lexicogrammatical features to register variables.

\section{5}

\section{Conclusion}

We have presented a study of online book reviews in English, Japanese and Chinese. Drawing from a corpus of 60 reviews of the novel "Q84" by Haruki Murakami (2O in each language), we provide an analysis of the generic structure of this emerging online genre. Our analysis shows that it develops in predictable stages, including Metapragmatic Comment (as preparation for the review, usually a background on the writer), Evaluation (of the book, the author, the plot, etc.) and Recommendation (whether the author endorses the book or not). At the same time, the texts in our corpus show some individual differences, in addition to differences across the three languages. Most notably, the Recommendation stage is most popular in English reviews, and is absent in Japanese reviews.

We postulate that the relative uniformity and order of the stages across the languages may be the result of constraints imposed by the online nature of the texts. It is plausible to assume that some of the writers on online book review sites are familiar with such reviews in other languages, especially in English, which is a dominant language on the Internet. This is certainly an open question, but one that should be explored: Do online genres converge more because of the ease of access to other languages online?

With respect to the rhetorical analysis of the reviews, we found that all three languages count Elaboration as the most frequent relation, often followed by Concession or Contrast, which serve to present alternative viewpoints to the opinion expressed by the author. Mapping generic stages to rhetorical relations, we observe that the texts are organised around the Evaluation stage, which 
is typically the nucleus of the entire review. While the rhetorical patterns are similar across the three languages, we observe some interesting cross-linguistic divergences in the lexicogrammar, particularly in the Recommendation stage. For example, English and Chinese tend to use expressions with modality that exert a high degree of obligation on the reader to carry out the recommended action. English favours commands that are directed toward the reader using imperatives e.g., 'Try him!' and Chinese prefers using explicit lexical wordings such as 建议 'recommend' from the writer's perspective. By contrast, instead of directly and explicitly recommending the book to the reader, Japanese reviewers tend to write about their personal feelings (cf. Fujimura-Wilson, 2017). Expressions of personal desire ほしい 'want' with a high degree of inclination toward the recommended action e.g. 絶対に買います 'I'll definitely buy it!' are examples of an indirect strategy adopted by Japanese reviewers. The general preference for indirectness in Japanese could be culturally motivated. However, we believe the selection of linguistic resources is also register-dependent, and the recommending context has brought this particular language feature to light through a contrastive analysis (cf. Chik, 2018; Matthiessen et al., 2008).

We conclude with an endorsement of the approach that we have undertaken here as a methodology to explore a new genre, and to do so across a number of languages. The top-down characterisation of generic stages allowed us to discover differences between the languages, while the RST approach (which can be seen as both top-down and bottom-up) revealed the different strategies for discourse organisation in this genre and in the three languages.

\section{References}

Blum-Kulka, Shoshana, Juliane House, and Gabriele Kasper (eds.). 1989. Cross-Cultural Pragmatics: Requests and Apologies. Norwood, NJ: Ablex.

Brown, Penelope, and Stephen Levinson. 1987 [1978]. Politeness: Some Universals in Language Usage. Cambridge: Cambridge University Press.

Carlson, Lynn, Daniel Marcu, and Mary Ellen Okurowski. 20o1. Building a discourse tagged corpus in the framework of Rhetorical Structure Theory. Proceedings of and SIGDIAL Workshop on Discourse and Dialogue, Eurospeech 2001. Aalborg, Denmark.

Cenni, Irene, and Patrick Goethals. 2017. Negative hotel reviews on TripAdvisor: A cross-linguistic analysis. Discourse, Context \& Media 16: 22-30.

Chik, Sonya. 2017. Constructing and shaping social reality across languages and cultures: a linguistic perspective [Paper Presentation].JASFL 2017 The 25th Anniversary Autumn Conference. Doshisha University, Kyoto. 
Chik, Sonya. 2018. Towards a systemic functional contrastive analysis of Japanese and English corporate legal discourse. In: Akila Sellami-Baklouti, and Lise Fontaine (eds.), Perspectives from Systemic Functional Linguistics. New York: Routledge, 182-205.

Chung, Cindy M., and Peter R. Darke. 20o6. The consumer as advocate: Self-relevance, culture, and word-of-mouth. Marketing Letters 17(4): 269-279.

Das, Debopam, and Maite Taboada. 2018. Signalling of coherence relations in discourse, beyond discourse markers. Discourse Processes 55 (8): 743-770.

De Jong, Ilona K. E., and Christian Burgers. 2013. Do consumer critics write differently from professional critics? A genre analysis of online film reviews. Discourse, Context \& Media 2(2): $75^{-83}$.

Fang, Hui, Jie Zhang, Yang Bao, and Qinghua Zhu. 2013. Towards effective online review systems in the Chinese context: A cross-cultural empirical study. Electronic Commerce Research and Applications 12(3): 208-220.

Feng, Wei, and Wei Ren. 2020. Impoliteness in negative online consumer reviews: A cross-language and cross-sector comparison. Intercultural Pragmatics 17(1): 1-25.

Fujimura-Wilson, Kayo. 2017. English and Japanese Compliments in Book Reviews of Academic Sociolinguistic Journals. 英語と英米文学 52: 1-26.

Goddard, Cliff, Taboada, Maite, and Trnavac, Radoslava. 2019. The semantics of evaluational adjectives: Perspectives from Natural Semantic Metalanguage and Appraisal. Functions of Language 26(3): 308-342.

Halliday, Michael A. K., and Ruqaiya Hasan. 1985. Language, Context, and Text: Aspects of Language in a Social-Semiotic Perspective. Oxford: Oxford University Press.

Halliday, Michael A. K., and Christian M. I. M. Matthiessen. 2014. Halliday's Introduction to Functional Grammar (4th Edition). New York: Routledge.

Hong, Yili, Ni Huang, Gordon Burtch, and Chunxiao Li. 2016. Culture, conformity and emotional suppression in online reviews. Journal of the Association for Information Systems 17(11): 737-758.

Hyland, Ken, and Giuliana Diani. 2009. Introduction: Academic evaluation and review genres. In: Ken Hyland, and Giuliana Diana (eds.), Academic Evaluation. Basingstoke, Hampshire: Palgrave Macmillan, 1-14.

Knott, Alistair, Jon Oberlander, Michael O'Donnell, and Chris Mellish. 20o1. Beyond elaboration: The interaction of relations and focus in coherent text. In: Ted Sanders, Joost Schilperoord, and Wilbert Spooren (eds.), Text Representation: Linguistic and Psycholinguistic Aspects. Amsterdam and Philadelphia:John Benjamins, 181-196.

Kranich, Svenja. 2016. Contrastive Pragmatics and Translation: Evaluation, epistemic modality and communicative styles in English and German. Amsterdam/ Philadelphia: John Benjamins Publishing Company.

Kunz, Kerstin, and Ekaterina Lapshinova-Koltunski. 2014. Cohesive conjunctions in English and German: Systemic contrasts and textual differences. In: Lieven 
Vandelanotte, Kristin Davidse, Caroline Gentens, and Ditte Kimps (eds.), Recent Advances in Corpus Linguistics: Developing and Exploiting Corpora. Amsterdam: Rodopi, 229-262.

Mackiewicz, Jo. 2008. Reviewer motivations, bias, and credibility in online reviews. In: Sigrid Kelsey, and Kirk St. Amant (eds.), Handbook of research on computer mediated communication. Pennsylvania/London: I g I Global, 252-266.

Mann, William C., and Sandra A. Thompson. 1988. Rhetorical structure theory: Toward a functional theory of text organization. Text 8(3): 243-281.

Martin, James R. 1984. Language, register and genre. In: Frances Christie (ed.), Children Writing: Reader. Geelong, Victoria: Deakin University Press, 21-30.

Martin, James R. and David Rose. 2008. Procedures and Procedural Recounts. Genre Relations: Mapping Culture. London: Equinox.

Martin, James R., and Peter R. White. 2005. The language of evaluation. Basingstoke, Hampshire: Palgrave Macmillan.

Matthiessen, Christian M. I. M. 2001. The environments of translation. In: Erich Steiner, and Colin Yallop (eds.), Exploring translation and multilingual text production: beyond content. Berlin, Germany: Walter de Gruyter, 41-124.

Matthiessen, Christian M. I. M. 2015. Register in the round: Registerial cartography. Functional Linguistics 2(9): 1-48.

Matthiessen, Christian M. I. M., and Kazuhiro Teruya. 2015. Registerial hybridity: indeterminacy among fields of activity. In: Donna Millar, and Paul Bayley (eds.), Permeable contexts and hybrid discourses. London: Equinox, 205-239.

Matthiessen, Christian M. I. M., Kazuhiro Teruya, and Wu Canzhong. 2008. Multilingual studies as a multi-dimensional space of interconnected language studies. In: Jonathan. J. Webster (ed.), Meaning in context: Strategies for implementing intelligent applications of language studies. London: Continuum, 146-220.

Moreno, Ana I., and Lorena Suárez. 2008. A study of critical attitude across English and Spanish academic book reviews. Journal of English for Academic Purposes 7(1): $15^{-26 .}$

Motta-Roth, Désirée. 1995. Rhetorical features and disciplinary cultures: a genre-based study of academic book reviews in linguistics, chemistry, and economics. (Doctoral Thesis), Universidade Federal de Santa Catarina.

Nakai, Fuki. 2002. The role of cultural influences in Japanese communication: A literature review on social and situational factors and Japanese indirectness. Intercultural communication research. Kanda University of International Studies Research Center (Ed.), (14): 99-122.

Ren, Wei. 2018. Mitigation in Chinese online consumer reviews. Discourse, Context \& Media 26: $5^{-12 .}$

Salmani Nodoushan, Mohammad A., and Hamed Montazeran. 2012. The Book Review Genre: A Structural Move Analysis. Online Submission 6(1): 1-30. 
Skalicky, Stephen. 2013. Was this analysis helpful? A genre analysis of the Amazon. com discourse community and its "most helpful" product reviews. Discourse, Context \& Media 2(2): 84-93.

Steiner, Erich. 2004. Translated texts: properties, variants, evaluations. Frankfurt: Peter Lang.

Steiner, Erich, and Colin Yallop (eds.). 2001. Exploring translation and multilingual text production: beyond content (Vol. 3). Berlin, Germany: Walter de Gruyter.

Swales, John M. 1990. Genre Analysis: English in Academic and Research Settings. Cambridge: Cambridge University Press.

Taboada, Maite. 2004. Building Coherence and Cohesion: Task-Oriented Dialogue in English and Spanish. Amsterdam and Philadelphia: John Benjamins.

Taboada, Maite. 2011. Stages in an online review genre. Text \& Talk-An Interdisciplinary Journal of Language, Discourse \& Communication Studies 31(2): 247-269.

Taboada, Maite, and Jack Grieve. 2004. Analyzing appraisal automatically. Paper presented at the Proceedings of AAAI Spring Symposium on Exploring Attitude and Affect in Text (AAAI Technical Re\# port ss\# O4\# O7), Stanford University, CA, pp. 158-161. AAAI Press.

Taboada, Maite, Marta Carretero, and Jennifer Hinnell. 2014. Loving and hating the movies in English, German and Spanish. Languages in Contrast 14(1): 127-161.

Thet, Tun Thura, Jin-Cheon Na, and Christopher S.G. Khoo. 2010. Aspect-based sentiment analysis of movie reviews on discussion boards. Journal of information science 36(6): 823-848.

Trnavac, Radoslava, and Maite Taboada. 2012. The contribution of nonveridical rhetorical relations to evaluation in discourse. Language Sciences 34(3): 301-318.

Trnavac, Radoslava, Debopam Das, and Maite Taboada. 2016. Discourse relations and evaluation. Corpora 11(2): 169-19o.

Tsujii, Koichi, Koji Tanaka, Yoshikatsu Fujita, and Kazuhiko Tsuda. 2017. Impression comments analysis in consideration of user profile of the online guests' review. Information Engineering Express 3(3): 21-30.

Turney, Peter D. 2002. Thumbs up or thumbs down?: semantic orientation applied to unsupervised classification of reviews. Paper presented at the Proceedings of the 4oth annual meeting on association for computational linguistics.

Upton, Thomas A., and Ulla Connor. 2001. Using computerized corpus analysis to investigate the textlinguistic discourse moves of a genre. English for Specific Purposes $20(4): 313-329$.

Vásquez, Camilla. 2014. The Discourse of Online Consumer Reviews. London: Bloomsbury. Virtanen, Tuija. 2017. Adaptability in online consumer reviews: Exploring genre dynamics and interactional choices. Journal of Pragmatics 116: 77-9o.

Zeldes, Amir. 2019. Multilayer Corpus Studies. New York: Routledge. 